\title{
Correction
}

\section{Correction: Marneweck et al., "Neural Representations of Sensorimotor Memory- and Digit Position-Based Load Force Adjustments Before the Onset of Dexterous Manipulation"}

In the article "Neural Representations of Sensorimotor Memory- and Digit Position-Based Load Force Adjustments Before the Onset of Dexterous Manipulation" by Michelle Marneweck, Deborah Barany, Marco Santello, and Scott Grafton, which appeared on pages 4724-4737 of the May 16, 2018 issue, the analyses reported for accounting for multiple comparisons is incorrect. The correct analysis used is as follows: Holm's Sequential Bonferroni Procedure (Holms, 1979). This correction does not affect the conclusions of the paper. The text has been corrected on the online PDF version.

The last sentence in the last paragraph of the Materials and Methods section should be replaced and be as follows:

All analyses report the adjusted false discovery rate using the Holm's Sequential Bonferroni Procedure (Holms, 1979).

In the References section, the following reference should be replaced:

Benjamini Y, Yekutieli D (2001) The control of the false discovery rate in multiple testing under dependency. The Annals of Statistics 29:1165-1188.

With this reference:

Holms, S (1979) A simple sequentially rejective multiple test procedure. Scand J Statist 6:65-70.

DOI:10.1523/JNEUROSCI.2053-18.2018 